\title{
Pressefreiheit in der Kirche oder: \\ Marginalien zur Deontologie der katholischen Journalisten
}

\author{
von Jesús Iribarren
}

An den Türmen alter Kirchen entdeckt man zuweilen noch Sonnenuhren. Manche erreichen eine erstaunliche technische Präzision: Auch in den schwierigsten Lagen war es dem Menschen gelungen, nach dem regelmäßigen Schattenwurf den genauen Zeitablauf zu messen. Aber man mußte sich bei diesen mit sinnreichen lateinischen Sprüchen ausgeschmückten Zifferblättern mit einer wesentlichen Beschränkung abfinden: Sie konnten den Durchgang der Sonne nur bei wolkenlosem Himmel anzeigen. Es waren "Uhren der glücklichen Stunden". Die Renaissance brachte einen Fortschritt: Auf den Glockentürmen erschienen die runden Zifferblätter der Pendeluhren und damit auch das Stundenläuten unabhängig von guten oder schlechten Zeiten, von erfreulichen oder unerfreulichen Anlässen.

Wenn Polemiken über die Frage der Informationsfreiheit in Gesellschaft und Kirche entstehen, so scheinen im Grunde genommen diese Auseinandersetzungen auf die Frage hinauszulaufen, ob man zur Sonnenuhr zurüdkkehren soll, ob der Zeitung verboten werden kann, ihrer Informationsaufgabe auch bei der Registrierung negativer Fakten nachzukommen? (Noch nie habe ich irgendeine Regierung gesehen, welche nach Zustimmung und Lob die Pressefreiheit bekämpfte). Ich möchte sofort beifügen, daß diese Vereinfachung - wie alle Vereinfachungen - eine Karikatur und nicht ein Porträt ist. Aber die Aussagekraft der Karikaturen beruht in der Regel auf der Ähnlichkeit mit dem Porträt.

\section{Anspruch auf Freibeit in jeder Presse}

Bevor wir feststellen können, daß die Zeitung, die wir gerade in der Hand halten, uns über religiöse Fragen orientiert, wissen wir, daß es sich um das Medium Zeitung handelt; daß diese Zeitung allein durch ihre Existenz und ihr „Zeitung-Sein “ uns vor ganz bestimmte Freiheitsansprüche stellt. Wir können uns daher nicht an ein Urteil über die religiöse Information wagen, bevor wir nicht das Problem der Information allgemein untersucht haben. Unbestreitbar ist in der Tat - als Wesensmerkmal - die Verpflichtung der Presse zu möglichst ausführlicher Information. Wie jede andere menschliche Freiheit darf diese grundsätzliche Aufgabe jeder Presse nicht ohne ernsthafte Berechtigung beeinträchtigt werden.

Msgr. Jesús Iribarren ist Generalsekretär der Union Catholique Internationale de la Presse (UCIP) in Paris. Er konzipierte das französische Original des vorliegenden Beitrags als Referat vor Mitgliedern des Instituts für Journalistik an der Universität Freiburg/Schweiz. CS will durch die Publikation dieser Ausführungen zum Problem der Pressefreiheit im katholischen Raum, die sich nicht in allen Punkten mit den Auffassungen der Herausgeber decken, zur Vorbereitung des Weltkongresses der UCIP im Juni 1971 in Luxemburg beitragen, der die „Offentliche Meinung in der Kirche“ zum Thema hat. 
Die Elektrizität war anfänglich ein Luxus und ein beschränktes Angebot einiger Produzenten. Heute ist der Stromverbrauch eine Notwendigkeit und ein selbstverständliches Allgemeingut geworden. Strom-Einschränkungen, zeitliche wie mengenmäßige, verursachen beim Verbraucher den gleichen Unwillen wie die Verletzung eines Rechtes, gleichgültig ob es sich beim Lieferanten um eine staatliche oder eine private Gesellschaft handelt.

Ahnlich verhält es sich mit der Presse. Der Leser fragt wenig nach dem Gründer der Zeitung und dessen ursprünglichen Zielen. Für ihn gehört es zur wesentlichen Aufgabe der Tageszeitung, über jedes interessante, auch geheim gehaltene Ereignis, so rasch wie möglich zu berichten. In dieser Erwartung kauft er sein Blatt. Der Leser duldet auf die Dauer weder Verspätungen noch Beschränkungen durch den Herausgeber, die Zensur oder irgendwelche pressure-groups. Scholastisch ausgedrückt würden wir sagen, daß zwischen "finis operantis" und "finis operis" (zwischen dem Mittel zum Erreichen des Zieles und dem Endziel) eine vollständige Trennung eingetreten sei; daß es den Leser, aufgrund der soziologischen Entwicklung wie auch der eigengesetzlichen psychologischen Beziehung Zeitung-Leser, wenig kümmert, welches Gesicht der Besitzer seiner Zeitung geben möchte. Wenn Geistliche sich krampfhaft bemühen zu "lehren“, während der Leser in erster Linie "informierr" sein möchte, herrscht Uneinigkeit über den Begriff, über Sinn und Aufgabe der Presse.

Die Presse- und Werbe-Agenturen haben die Nachricht als Kaufangebot an Millionen von Lesern endgültig zur Ware gestempelt. Der Käufer bestimmt Eigenschaften und genaues Gewicht der von ihm gewünschten Ware, wie bei Möbeln und Nahrungsmitteln, unbekümmert darum, mit welchen Eigenschaften und zu welchem Gewicht andere - zivile oder kirchliche Behörden zum Beispiel - verkaufen oder unterschlagen möchten. Das will nicht heißen, daß die Regierung, der Bischof oder der Zeitungsbesitzer die Zeitung nicht benützen können, um bekanntzugeben, was sie persönlich denken oder als notwendig erachten. Auch das interessiert den Leser; denn es ist ein Teil des universellen und aktuellen Angebots. Die geistige Verwandtschaft mit einer Ideologie, die Zugehörigkeit zu einer Organisation oder einer Kirche bewirken, daß gewisse Leser in ihrer Zeitung die offizielle Meinung suchen, welche sie in ihren Überzeugungen bestärkt und für die aktive Mitarbeit in ihrem Milieu richtungweisend ist. Entsteht jedoch beim Leser der Verdacht, daß die Zeitung ihm wesentliche Informationen vorenthält, nur oder gerade weil sie den Auffassungen des Verlegers oder ihm nahestehender Kreise widersprechen, wird der Leser das Blatt unter Hinweis auf manipulierende Praktiken aufgeben. Hier liegt wohl der Grund für den Auflagenschwund zahlreicher apologetischer Regierungs- und Gruppenzeitungen. Das Publikum weiß wohl zu unterscheiden zwischen "public relations" und "Propaganda" einerseits, welche jenem zugutekommen, der sie in Auftrag gibt und bezahlt, sowie der Presse-Publizistik andererseits, die der Offentlichkeit dient und wegen dieser Dienstleistung gekauft wird.

b) Die Informationsfreiheit entspricht einem Bedürfnis der Gesellschaft

In der modernen Gesellschaft, in welcher der Mensch von seinen traditionellen Wurzeln losgerissen ist, hat die Information die Orientierungsaufgabe übernommen, 
welche früher der Tradition zukam. Ich möchte die Aussage mit einem Bild verdeutlichen. Der Hirt, der Krieger und der Kutscher verfügten über feste Orientierungspunkte: Berge, Flüsse, Kirchtürme. Der Pilot eines Flugzeuges kann sich nicht auf einen solchen festen und vertrauten Orientierungspunkt verlassen. Er bedarf einer ständigen Funkverbindung, die ihn laufend über die unsichtbaren und ständig wechselnden Koordinaten seines Fluges informiert.

Damit soll das Uberleben einer Tradition in der mobilen Welt von heute nicht verneint werden. Die Uberlieferung existiert, gewiß, aber nur in den Büchern, wahrnehmbar allein noch für eine gebildete Minderheit oder eine weniger urbanisierte Bevölkerung. Für den Menschen im Wolkenkratzer, den Kumpel im Stollen, den Emigranten, den Metro-Passagier, kann nur das Radar der Zeitungen sagen, wo heute morgen Politik, Religion, öffentliche Moral, die sozialen Konflikte, der Sport stehen.

Genauso verhält es sich für die Kirche. Was bedeutet noch „christliche Tradition“ dort, wo nach der Statistik zehn oder fünf Prozent der Erwachsenen am Gottesdienst teilnehmen? Die einzige Orientierungsmöglichkeit, welche dem auf seine eigenen Wahrnehmungen angewiesenen Menschen erlaubt, seinem Leben einen Sinn zu geben und eine moralische Haltung einzunehmen, ist jene, welche ihm von Tag zu Tag durch die verschiedenen Informationskanäle - sich selbst wiedersprechend - zukommt. Die Konversion der Prinzessin Irene von Holland vor ihrer Heirat, der Tod des Guerilla-Priesters Camillo Torres, die Heirat Jacqueline Onassis', der Feuertod des jungen Tschechen Jan Palach, die Zusammenkünfte revolutionärer Priestergruppen; alle diese Ereignisse werden vom Mann der Straße nicht nach den Kriterien einer seit dem Mittelalter destillierten christlichen Moral beurteilt, sondern nach Grundsätzen, die in den Balkentiteln seiner Zeitung und einer um zwei Uhr morgens in aller Hast redigierten Meldung ihren Niederschlag finden. Diesem „Mann der Straße" heute eine genügend breite Information zu bieten, damit er selbst urteilen und persönliche Entscheidungen fällen kann, ist für die Kirche eine Verpflichtung.

\section{c) Eine informations-schwache Zeitung ist nicht lebensfähig}

Eine möglichst breite und vertiefende Information entspricht heute nicht nur einer öffentlichen Aufgabe und ist sozial wichtig, sondern stellt auch eine wirtschaftliche Notwendigkeit dar. Ein Massenkommunikationsmittel, das dieser Notwendigkeit nicht Rechnung trägt, beschränkt die eigene Lebensfähigkeit. Weil sie bis zu 80 oder mehr Prozent der Zeitungskosten decken, sind Werbe- und Anzeigen-Agenturen zu Entscheidungs-Trägern über Leben und Tod von Zeitungen geworden. ${ }^{1}$ Der zwangsmäßige Kreislauf: Werbung proportional zur Auflage, Auflage proportional zum Publikums-Interesse, Publikumsinteresse proportional zur Befriedigung des Neuigkeiten-Bedürfnisses. Informieren oder sterben, eine andere Alternative gibt es nicht.

Eine kirchliche Behörde, welche die Informationsfreiheit beschränkt, kann die Existenz ihrer Zeitung retten, indem sie das Defizit, das die Inserateneinnahmen gedeckt hätten, durch Subventionen ausgleicht. Sie kann aber nicht verhindern, daß die Leser trotzdem zu anderen Zeitungen greifen, von denen sie informiert werden. Mit anderen Worten: Die kirchliche Behörde kann einen Titel auf unbestimmte Zeit hinaus stützen, aber es wird nicht eine das pulsierende Leben der Straße widerspiegelnde Zeitung sein, sondern ein einbalsamierter Leichnam. 
d) Im Konkurrenzkampf mit den informierenden Zeitungen kann die in ihrer Informationsf reiheit beschränkte Zeitung nicht überleben

Die Entscheidung zum Verzicht auf eine schlecht informierende Zeitung wird erleichtert durch die Gewißheit, daß es andere Blätter gibt, die regelmäßig informieren, und dies mit einer bemerkenswerten Objektivität. Auch der Umstand, daß diese Presse oft einen einseitigen oder gar feindseligen Standpunkt zur kirchlichen Lehre einnimmt, ändert daran nichts; denn es handelt sich um eine "ergänzende Parteilichkeit". Verschiedene Parteilichkeiten ergeben die Wahrheit. Es wird kaum jemand bezweifeln wollen, daß Millionen von Katholiken sich aus Zeitungen und Zeitschriften informieren, die keineswegs kirchenfreundlich eingestellt sind. Es wäre illusorisch, sie daran $\mathrm{zu}$ hindern. Ihnen in der konfessionellen Presse vorzuenthalten, was sie in der neutralen Presse lesen können, ist zwecklos und bedeutet nicht mehr und nicht weniger als einen langsamen Verlust an Prestige. ${ }^{2}$

Zahlreich sind die Männer der Kirche, welche versichern, ihr Möglichstes zur Förderung der katholischen Presse getan zu haben. Genau besehen machen sie das Gegenteil. Sie tun alles, um die Emigration der Leser zur säkularen Presse zu beschleunigen. Es geht sogar noch weiter. Wie oft kam es in den letzten Jahren vor, daß Dokumente und Tatsachen, welche die kirchlichen Behörden geheimhalten wollten, mit großen Auflagen und erheblichem Wirbel unter ein interessiertes Publikum gebracht wurden. (Auf die Veröffentlichung solcher geheimzuhaltender Dokumente und Tatsachen durch Katholiken in katholischen Zeitschriften werde ich noch zurückkommen.) In solchen Fällen steht eines fest: Verlierer ist immer jene Zeitung, die glaubte, eine solche Information nicht bringen zu können, während andere sie breitschlugen. Es geht mir hier nicht darum, den moralischen Wert solcher Situationen zu beurteilen. Ich möchte nur die Schlappe für die Geheimhaltungspolitik einerseits und deren wirtschaftliche Folgen im Konkurrenzkampf anderseits unterstreichen.

Die Diversität der Kriterien und der soziologischen Situation der Kirche in den verschiedenen Ländern bringt es mit sich, daß die konfessionelle Presse Deutschlands, Italiens, Hollands, Spaniens, Frankreichs, Kolumbiens oder der Vereinigten Staaten ihre Information recht verschieden gestaltet. Die Nachrichten-Agenturen, die Hörfunksendungen in fremder Sprache - die Fremdsprachenkenntnisse nehmen in gebildeten Kreisen stetig zu - bemühen sich um möglichst freiheitliche weltweite Verbreitung der Informationen, so daß irgendwelche informationshemmenden Maßnahmen der Regierungen und der Kirche im Endergebnis wirkungslos bleiben müssen.

\section{Erfordernisse für eine freibeitliche Information in der Kirche und über die Kirche}

Angesichts der schon dargelegten Gründe und der für unsere Zeit nicht mehr wegzudenkenden Allgegenwart der Massenkommunikationsmittel kann die Kirche sich den der Welt der Information eigenen Gesetzen nicht entziehen. Wenn sie nicht selbst informiert, - und dadurch bewirkt sie die Lähmung, den Mißkredit und das Verschwinden ihrer eigenen Presse - , werden andere diese Aufgabe übernehmen. Alle besonders schwierigen Fragen, die man bei den Konzilsbesprechungen aufzuwerfen vermied - unter anderem, damit sie nicht Opfer der "Sensationspresse“ werden 
konnten - finden sich heute, zuweilen ebenso leichtfertig wie verworren präsentiert, im Themen-Angebot der Presse aller Seriösitäts-Grade. Aber sind der von außen kommende Druck und die Einbeziehung in eine soziologische Entwidklung, welche der Kirche im Grunde unangenehm oder ihrem Wesen gar widersprechend ist, wirklich die einzige Berechtigung zur Information über die Kirche?

\section{a) Die Kirche verteidigt die Freiheit}

Mit Nachdruck können wir die letzte Frage verneinen; denn wir sind im Gegenteil überzeugt davon, daß die Informationsfreiheit in der Kirche logischer wäre als irgendwo sonst. Der Kampf für die Freiheit des Menschen ist eine der großen und tiefen Konstanten in der Kirchengeschichte; denn für die Kirche ist die Freiheit des einzelnen ein Spiegelbild Gottes in ihm, da jeder ja nach Seinem Bilde geschaffen wurde. Dem sachlichen Kritiker und Kirchen-Historiker zeigen sich zwei nebeneinander verlaufende Entwicklungslinien. Die mehr oberflächliche weist gelegentlich offensichtliche Verletzungen der Freiheit oder empörende und schmerzliche freiheitsfeindliche Haltungen aus: Man denke an die Inquisition, die erzwungenen Konversionen und - im 19. und am Anfang des 20. Jahrhunderts - den Mangel an Verständnis für die Gewerkschaftsbewegung und den politischen Liberalismus. Die andere, tiefer liegende Linie in der Geschichte der Kirche bezeugt dem Beobachter jedoch, daß selbst in vordergründig freiheitsfeindlichen Zeiten, freiheitsfeindlich sogar in der Haltung von Kirchenmännern und Päpsten, die Kirche als solche vehement die verletzte Freiheit verteidigt hat. Man denke dabei an die nie widersprochenen Freiheiten des Glaubensbekenntnisses und des Eintrittes in die Kirche, wie auch an den mit aller Energie bekämpften Totalitarismus. Der objektive Historiker wird anerkennen müssen, daß von diesen beiden Linien in der Kirchengeschichte die tiefere auch die weit wesentlichere und beständigere war als die oberflächliche. Die hieraus für die Informationsfreiheit zu ziehenden Konsequenzen sind klar. Ich beschränke mich im folgenden auf einige Klarstellungen, fast schematisch, und beginne mit den augenfälligsten Aspekten.

b) Die offizielle Doktrin über die Meinungs- und Ausdrucksfreiheit

Wenn Paul VI. - die Doktrin von „Pacem in terris“ bestärkend - erklärt, daß „eine der bemerkenswertesten Errungenschaften des modernen Menschen die - in gewissen Ländern vorläufig leider nur mündliche - Anerkennung der freien Meinungsäußerung und Meinungsbildung, sowohl der Gruppe wie des Individuums, ist" ${ }^{\text {"3 }}$, so unterstreicht diese Äußerung auch die Legitimität der freien Meinungsäußerung und Meinungsbildung im kirchlichen Raum. Innerhalb der Kirche Rechte zu verweigern, die man von der profanen Gesellschaft fordert, wäre unredlich und unkorrekt. Die Kirche ist eine Institution öffentlichen Rechts: Wenn die Staaten als unwiderruflich anerkennen, daß die Handlungen der Behörden und die Wechselfälle des politischen Lebens dem Volke zur Kenntnis zu bringen sind, hat diese Unwiderruflichkeit auch für die Kirche Geltung. Auch für die Kirche gilt der von „Pacem in terris" proklamierte Grundsatz, daß jeder Mensch ein universelles, unverletzliches und unveräußerliches Recht auf "objektive Information in öffentlichen Angelegenheiten $^{{ }^{4}}$ habe. 
Die Information gehört zum Allgemeinwohl: Die Autorität - auch die religiöse hat die Pflicht, dieses Allgemeinwohl zu fördern und nicht zu beeinträchtigen. Wenn Gründe zur Beschränkung dieses Gutes sich ergeben, müssen sie berechtigt sein; im Zweifel fällt die Entscheidung immer zugunsten der Freiheit.

\section{d) Mangelnde Information führt zur Passivität}

Fehlende Information der Offentlichkeit führt zur Passivität. Es ist nicht schwer, die Ursache der erschreckenden Passivität eines Großteils der Katholiken auszumachen. Die fehlende "Evangelisierung “ oder das Fehlen einer Verkündigung der "Guten Nachricht" bedeutet in vielen Fällen nichts anderes als Mangel an Nachrichten.

\section{e) Ausdruck des "Sensus fidelium“}

Bei der Definition des übernatürlichen Wesens der Kirche stellen wir fest, daß der "Sensus fidelium “, die Meinung des Gottesvolkes, eine wichtige Rolle spielt bei der Führung der Kirche durch den Heiligen Geist. Die Hierarchie darf diese wichtige Stimme der Gläubigen nicht überhören. Die modernen Massenmedien sind Träger dieser öffentlichen Meinung.

\section{f) Notwendigkeit des Dialogs}

Nur während der Zeit ihrer Minderjährigkeit spricht ein Vater im Namen der Kinder. Volljährige Kinder haben das Recht, ihre Meinung selbst auszudrücken. In der Kirche ist das nicht anders. Familie setzt den Dialog voraus. Uberall dort, wo der Dialog zu zwei parallelen Monologen verkümmert, ist das Weiterbestehen der Familie gefährdet. Ebensolche parallele Monologe sind oft die Predigt und der Zeitungsartikel. Die Kirche unterhält verschiedene Formen von Dialog: Dialog Kirche-Welt, Dialog zwischen den Kirchen, Dialog mit den nichtchristlichen Religionen, Dialog mit den Nicht-Glaubenden. Es wäre deshalb absurd, wenn zwischen den Gläubigen und der Hierarchie nicht auch ein Dialog existieren würde, der heute über die Massenmedien geführt wird, was andere Dialogmöglichkeiten, individuelle und private, nicht ausschließt.

\section{g) Legitime Meinungs-Pluralität}

Eines der Resultate des Dialoges innerhalb der Kirche ist die Tatsache einer Diversität von Meinungen, von gelegentlich sogar gegensätzlichen Meinungen. Die Meinungsfreiheit steht nicht im Gegensatz zum Gehorsam und zur Einheit. Es gibt eine legitime Pluralität der Völker, der Kulturen, der Riten. Es gibt sie auch bei den Episkopaten, den Theologen. Es ist nicht mehr als logisch, daß sie auch bei den Gläubigen vorhanden ist und sich in den Zeitungen widerspiegelt. 


\section{h) Gegen die Zentralisierung}

Vielfalt wirkt befruchtend und anregend auf das schöpferische Wirken und auf den Fortschritt. Sie ist gleichzeitig auch von Bedeutung zur Uberwindung der entwicklungshemmenden Zentralisierungstendenzen, wie sie in jeder menschlichen Gesellschaft vorkommen. Es gibt Bürokraten, die jegliche Vielfalt verabscheuen. Sie wünschen sich Bäume, die nur aus Stämmen bestehen, ohne diese „anarchistische" Neigung, zu vielfältige und vom Hauptstamm zu weit „entfernte “ Aste und Zweige zu treiben. Sind nicht gerade die von der Mitte am weitesten entfernten Aste immer $Z_{w}$ eige der stärksten, schönsten und unsichtbar mit der entfernten Wurzel vereinten Bäume? Die Wurzel in der Kirche ist Christus, mit dem Papst und den Bischöfen. Wenn die Einheit gewahrt bleibt, ist die "Entfernung" nur scheinbar. Sie ist ein Zeichen von Vitalität.

\section{i) Das Negative aufzeigen}

Dialog und Meinungsfreiheit führen, in schwerwiegenden Fällen, auch zur Offenlegung der negativen Aspekte. Mißbräuche um jeden Preis mit dem Mantel des Schweigens zu verdecken, bewirkt den Eindruck, als wolle man, wenn nicht die Fehlenden, so doch die Fehler in Schutz nehmen: Es sieht aus wie das Eingeständnis der Mitschuld. Früher oder später wird ein Skandal doch aufgedeckt, und das Ergebnis ist Mißkredit für die Kirche und Anklage der Heuchelei. Damit die Publikation ein Dienst an der Sache Gottes und nicht eine "verachtenswerte Handlung einer antiklerikalen Sensationsjournalistik " ist, muß eine Bedingung erfüllt sein: der Ton, die Zurückhaltung, die unmißverständliche Treue zur Kirche müssen als Beweggrund erkennbar werden. Wenn dies alles fehlt und wenn eine Publikation „sich auf das Negative spezialisiert", so wäre dies keine prophetische Denunziation, sondern schadenfrohes Spionieren.

Aufzeigen des Negativen bezweckt etwas anderes: die Behörden anzuspornen und jene Maßnahmen zu veranlassen, welche die eingeschlagene Richtung korrigieren. Die Geschichte der sozialen Bewegungen zeigt, daß es ohne Streiks keine Lohnerhöhungen gegeben hätte. Zu sagen, die Kirche „komme spät“, ist ein Gemeinplatz. Ohne das Aufzeigen der Übel käme sie noch viel später. Die Presse braucht die notwendige Freiheit, um den Männern der Kirche zu helfen, sich selbst zu korrigieren und neue Ziele zu setzen.

\section{j) Keine zerstörende Kritik}

Die Betonung liegt auf „helfen“. Bittere Kritik, „Forderungen“ und nachtragende Vorwürfe helfen nicht. Es gilt, eine Gewissenerforschung zu halten, sowohl seitens der als integralistisch wie auch seitens der als progressistisch bezeichneten Presse und auch jener Presse, welche in der Mitte zwischen diesen Richtungen steht. Die sprachlichen Ubertreibungen und „essigsauern" Anklagen kommen zu häufig vor und sind keineswegs das Monopol einer einzigen Gruppe.

\section{k) Die Grenzen des Secretum}

Aus Angst vor dem Skandal oder auf der Suche nach einem wirkungsfähigeren Regierungsstil haben Gruppen und Personen in der Kirche das Geheimnis besonders zu pflegen versucht. Ich bin der festen Ubberzeugung, daß dem Geheimnis in Gesell- 
schaft und Kirche immer ein besonderer Platz zugekommen ist, noch zukommt und auch in Zukunft zukommen wird: ob es sich nun um das sakramentale oder berufliche Geheimnis handelt, um das loyal versprochene oder um jenes, das durch gewöhnliche Vorsicht geboten ist. ${ }^{5}$

Darüber hinaus läßt sich eine Geheimnis-Neurose jedoch nicht rechtfertigen, vor allem nicht in den drei nachfolgend beschriebenen Fällen: Wenn die Gläubigen ein "Recht auf Kenntnis" haben; wenn die Vorteile der Information größer sind als jene der Geheimhaltung (ein Problem der Klugheit für den Regierenden); oder wenn die Geheimhaltung sich als unmöglich erweisen muß. Unmögliche Pflicht ist keine Pflicht, in jeder vernünftigen Moral. Die Erfahrungen der letzten zehn Jahre mit der Weltpresse sprechen für sich. Alle des Lateinischen kundigen Journalisten, sogar jene, welche diese Sprache nicht beherrschten, konnten während des Konzils jederzeit über sämtliche Unterlagen verfügen. Die Photokopien der Dokumente, einmal sogar eine wörtliche Wiedergabe, trugen stets den Vermerk "sub secreto“ (= geheim). Man hatte dabei den Eindruck, als ob man an einem Tag mit strömendem Regen den Wetterbericht als geheim bezeichnen wollte. Dieses Geheimnis in einer Auflage von 100000 Exemplaren wird sich wohl kaum wiederholen. Die Gewissen sind von Tag zu Tag überzeugter, daß ein Geheimnis, welches Personen oder Allgemeinwohl schützt, seine Berechtigung hat, gewisse willkürliche Geheimhaltungen jedoch nur Angst, Unsicherheit oder den Wunsch nach unbeschränkter Machtausübung zum Ausdruck bringen. ${ }^{\circ}$

Trotzdem: Der Wunsch nach einer informationsfreundlichen Haltung der Kirche kann keineswegs offensichtliche Mißbräuche der letzten Jahre rechtfertigen: Durchsickern von Dokumenten, welche nie den Weg in die Offentlichkeit hätten nehmen sollen, auf dem sie lanciert wurden. Ein Journalist, der versprochen hat, über eine vertrauliche Information $\mathrm{zu}$ schweigen, muß dieses Versprechen halten, auch wenn er Gefahr läuft, wegen dieses Schweigens seine Stelle zu verlieren. Noch stärker ist an die Schweigepflicht gebunden, wer als Mitglied einer Kommission hierzu durch Eid verpflichtet ist oder ein Schreiber, der seine erste Seite mit der unsympathischen Formel „sub secreto" versehen muß. Wenn ihm dies unsympathisch ist, hat er ja die Möglichkeit, dem $\mathrm{Zwang}$ aus dem Wege zu gehen und seine Stelle zu verlassen.

Damit soll keineswegs gesagt sein, daß die Presse immer schuldig wird, wenn sie der Offentlichkeit ein Dokument zur Kenntnis bringt, das seine Autoren geheimhalten wollten. Entscheidend sind die Berechtigung der Geheimhaltung und die Art und Weise, wie ein solches Dokument in die Spalten der Zeitung gelangt ist. Man hat guten Grund zu der Annahme, daß gewisse Kleriker und subalterne Beamte sektiererischen Eifer über ihre Berufsehre gestellt haben, wenn sie ihrer Pflicht untreu wurden und der Presse diese Untreue käuflich anboten; denn der Journalismus ist ein ehrlicher Beruf.

\section{Die Grenzen der Freibeit gegenüber der Doktrin und der Autorität der Hierarcbie}

\section{a) Das Dogma, unübersteigbare Grenze}

Ich glaube nicht, die Forderungen nach Freiheit beschnitten $z u$ haben. Aber eine Freiheit ohne Grenzen zerstört sich selbst. Unbeschränkt frei ist weder der Mathematiker, noch der Historiker, noch der Politiker, noch der Journalist. Eine erste - 
und nicht überschreitbare - Grenze für die Informationstätigkeit des katholischen Journalisten ergibt sich aus der Unveränderlichkeit der Dogmen. In den säkularen Gesellschaften ist die Autorität an die Gerichtsbarkeit gebunden. Sie zielt aufs Allgemeinwohl und manifestiert sich in Regierungsmaßnabmen. Eine politische Gesellschaft, welche ihren Mitgliedern Ideen aufzwingen will, ist eine Tyrannei und kann höchstens eine äußerliche Zustimmung erreichen. In der Kirche Christi gibt es jedoch eine doktrinale Autorität, deren Ziel die Wabrbeit ist und welche die innere Zustimmung zu Glaubenssätzen voraussetzt.

Man kann dieser Glaubensgemeinschaft angehören oder nicht. Wer ihr angehört, hat die als unfehlbar promulgierten Definitionen als Wahrheiten anzunehmen. In der Logik des katholischen Journalisten ist die Ablehnung eines Dogmas nicht in erster Linie eine - infolge der Exkommunikation schwerwiegende - rebellische Stellungnahme und Sünde, sondern vor allem eine falsche Stellungnahme. Die Grenzen der Wahrheit sind die Grenzen unserer Meinungsfreiheit und ihrer Darstellung. Dieser Grundsatz findet heute ständige Anwendung bei der Behandlung der Information über theologische Fragen, die Gegenstand von Presse-Polemiken sind oder mit ökumenischen Begriffen dargestellt werden. Den Katholiken die ihnen fernstehenden Glaubenslehren darzulegen, ist immer zulässig und journalistisch wertvoll. Der Verneinung zuzustimmen oder gar teilzunehmen an der In-Frage-Stellung von Dogmen, ist hingegen gleichbedeutend mit Annahme des Irrtums.

\section{b) Das ordentliche Lehramt ist nicht unfehlbar}

Das ordentliche Lehramt der Päpste und der Kirche steht rangmäßig unter dem feierlichen, endgültigen Lehramt. Eindeutige Irrtümer des ordentlichen Lehramtes früherer Zeitepochen, hervorgegangen aus bestimmten Kultur- und Milieu-Einflüssen, die nicht ein so klares Urteil wie in späteren Epochen erlaubten, sind durch die Kirche anerkannt und korrigiert worden.

Niemand verteidigt heute mehr die "weltliche Vorherrschaft" der Päpste über die Könige, welche das Mittelalter anerkannte und Bonifaz VIII. in seiner Bulle „Unam Sanctam" sehr autoritär proklamierte. Sie hat sich als falsch erwiesen.

Vom Pontifikat Innozenz' XII. bis zu jenem von Benedikt XIV. dauerte die Kontroverse über die "chinesischen Riten“, infolge der Unnachgiebigkeit von sechs Päpsten (Innozenz XII., Clemens XI., Innozenz XIII., Benedikt XIII., Clemens XII. und Benedikt XIV.) und des Heiligen Offiziums. Jeglicher Widerstand wurde im Jahre 1742 unterdrückt. Im Jahre 1935 korrigierte Pius XI. nicht nur die Stellungnahme, sondern auch die Doktrin.

Die während des ganzen Mittelalters unbestrittene Lehre über den "Wucher" - man denke an das Sprichwort: pecunia non parit - hätte angepaßt werden müssen, als sich im 15. Jahrhundert die Handelsbräuche und damit auch die Natur des Geldes änderten. Aber noch mitten im 18. Jahrhundert hielt Benedikt XIV. mit seiner Enzyklika „Vix pervenit“ sehr streng an der alten Lehre fest, obwohl deren Anwendung schon seit zwei Jahrhunderten angesichts der veränderten Verhältnisse ein offensichtlicher Irrtum war. Die katholische Morallehre betrachtet das verzinsbare Darlehen heute als eine Selbstverständlichkeit. - Die Kirche erkennt an, daß das Vorhandensein solcher Irrtümer in der Vergangenheit auch deren Möglichkeit in der Zukunft nicht ausschließt. 
aa) Nicht in Frage stellen

Darf nun der Schluß gezogen werden, daß Katholiken über alle Lehren diskutieren können, welche nicht ausdrücklich von Papst und Konzil unter Berufung auf das von Gott stammende Vorrecht der Unfehlbarkeit verkündet wurden? - Es gibt zwei wichtige Beweggründe, die uns veranlassen, sowohl innerlich wie äußerlich den Verkündigungen des ordentlichen Lehramtes beizupflichten. Der erste Beweggrund ist rein menschlicher Natur. Seit 20 Jahrhunderten haben die Päpste die Wahrheit und das geistige Gut der Gesellschaft vor vielerlei Irrtïmern und Angreifern beschützt, die auch unter sich keineswegs einig waren. Die Wissenschaftler mußten weit öfters als die Päpste ihre Stellungnahmen berichtigen. Der Pendelschlag zwischen Tyrannei und Revolution in der Geschichte ist ein konstanter Beweis für die Unzulänglichkeit der politischen Philosophien. Als Gesamtheit betrachtet war die Lehre der Kirche in nicht-dogmatischen Fragen - im Verlaufe dieser 20 Jahrhunderte geradlinig. - Der zweite Beweggrund ist ein übernatürlicher: Katholiken glauben, daß Christus ständig, auch durch die Vermittlung fehlbarer Menschen, seine Kirche regiert. Der Spielraum von Vertrauen, der jenen Menschen zugestanden werden muß, ist jener, den wir Christus schulden.

\section{bb) Hilfe für das Lehramt}

Doch das grundlegende Prinzip bleibt bestehen: Das ordentliche Lehramt ist nicht unfehlbar. Die Verpflichtung, die Erklärungen dieses Lehramtes jederzeit zu respektieren, könnte - zumindest theoretisch - eine Pflicht werden, mit ehrfürchtigem Schweigen etwas als richtig anzuerkennen, das de facto falsch ist, eine paradoxale Pflicht. In dieser Situation befanden sich oft Männer der Kirche, welchen Exkommunikation oder schwere doktrinale oder disziplinarische Verurteilung drohte.

Für Grenzfälle bieten Theologie und Sittenlehre wertvolle Kriterien. Schweigen ist in solchen Fällen nicht ein inaktives Schweigen. Im Ausmaß ihrer Lehr-Autorität, ihrer Kenntnis der Doktrin und der verschiedenen Aspekte der debattierten Frage, haben die Bischöfe, die Theologen und die Experten das Recht und die Pflicht, ihre gegenteiligen Argumente vorzubringen und zur Erhellung des Problems beizutragen, bis das Lehramt die erforderliche Berichtigung vornimmt. Dies sollte unter zwei Bedingungen geschehen: den Skandal eines scheinbaren Ungehorsams vermeiden sowie wirklich über ernstzunehmende Gründe und klare Argumente verfügen, um der offiziellen Doktrin widersprechen zu können. Ohne diese Voraussetzungen würde der Weg zur Anarchie der freien Stellungnahme durch inkompetente Kritikaster geöffnet, wie auch zur Ablehnung der Lehr-Autorität in der Kirche. Man muß sich bewußt sein, daß es verhältnismäßig leicht ist, Grenzfälle auf alle Fälle zu übertragen und die Möglichkeit des Irrtums vorzubringen, um den Irrtum der Autorität wie das Recht des Untergebenen zum Widerstand zu behaupten.

\section{cc) Durch das Mittel einer ausgewogenen Information}

Die Situation der Presse ist ohne Zweifel sehr komplex, weil es ihr durchaus möglich ist, Millionen von Menschen mit Skandalen zu konfrontieren. Anderseits - und das liegt sozusagen in der Natur der Massenmedien - werden die differierenden Meinungen der Bischöfe, Theologen und Experten immer den Weg bis in die Spalten der Zeitungen finden. Diese zwei an sich nicht wünschenswerten Sachverhalte kann der Journalist nicht aus der Welt schaffen. 
Schweigen ist keine Lösung. Was für einen Zweck hätte z. B. im Fall der Enzyklika "Humanae vitae" ein Schweigen der katholischen Journalisten inmitten eines weltweiten Wirbels von Protesten und unterschiedlichsten Kommentaren? Auch offensidhtliche Differenzen kann man nicht verschweigen. Im Falle der genannten Enzyklika wurden heftige Angriffe gegen den "Osservatore Romano" geführt. Beobachter der Szene behaupteten, diese Zeitung veröffentliche nur zustimmende Stellungnahmen und begnüge sich mit Andeutungen für gegensätzliche Stimmen.

Die richtige Lösung besteht meines Erachtens in einer umfassenden, aber ausgewogenen Information. Damit meine ich eine Information, die grundsätzlich auf der Seite der Autorität steht (der doktrinalen oder disziplinarischen) und deren Urteile man annimmt in ihrer Wertung, solange nicht das ausgesprochene Gegenteil beweisbar wird. Gemeint ist eine Information, welche die exakte Bedeutung der Widersprechenden und des Widerspruches weitergibt, ohne einseitig zum Sprachrohr von Personen und parteiisch fixierten Meinungen zu werden, Stellungnahmen, welche objektiv besehen nur das Gewicht haben, welches ihnen die Presse künstlich gegeben hat. Anderseits aber dürften die soliden Gründe jener nicht verschwiegen werden, die für ihre abweichenden Auffassungen ernstzunehmende Motive vorbringen können. Eine Information, die nicht als rebellisch solche Standpunkte darstellt, welche den Standpunkt des Lehramtes eher vervollständigen als widerlegen, und die mehr aufbauen als opponieren will.

Eine solch ausgewogene Information kann nicht bieten, wer die diskutierten Probleme nicht gründlich beherrscht. Eine entsprechende Ausbildung ist notwendig, um über religiöse wie auch über wirtschaftliche oder wissenschaftliche Fragen informieren zu können. Wem diese Ausbildung abgeht, der muß die Selbstverleugnung aufbringen, um an kompetenter Stelle Rat zu holen.

\section{c) Für die freie Diskussion offener Fragen}

Es braucht wohl kaum speziell betont zu werden, daß der katholische Journalist in allen weltlichen und religiösen Fragen, welche der freien Diskussion offenstehen, Meinungen vertreten kann, die nicht mit den Auffassungen seines Bischofs übereinstimmen, und $\mathrm{da} ß$ er diese Meinungen überall zum Ausdruck bringen kann. Von dieser Voraussetzung ist jedenfalls auszugehen.

\section{d) Autonomie der Redaktion in weltlichen Fragen auch in kirchen-abhängigen Zeitungen}

aa) Damit die Kirche nicht kompromittiert sei

Die Meinungsfreiheit der Redaktion einer ausdrücklich als katholisch betitelten Zeitung, oder eines Blattes, das Eigentum einer kirchlichen Institution ist, mag weniger klar sein. Es ist jedoch für die Hierarchie von größtem Interesse, daß sie dem Chefredakteur üblicherweise einen breiten Spielraum an Selbständigkeit läßt. Päpste und Bischöfe sind unter bestimmten Umständen zu großer Wortkargheit gezwungen. Es mangelt nicht an Beispielen: Denken wir an Pius XI. im Italien Mussolinis, an Pius' XII. mühevolle Versuche, eine unparteiische Haltung gegenüber den kriegführenden Staaten Deutschland, Rußland und USA zu bewahren; an die Bischofs- 
konferenzen in Ländern mit Diktatur-Herrschaft oder Rassendiskriminierung. Jedes päpstliche oder bischöfliche Wort wird in solchen Ländern für oder gegen das herrschende Regime politisiert. "Velis nolis" — ob sie will oder nicht, die Hierarchie wird in eine Parteistellung gezwungen.

Unter solchen Umständen könnte die Presse Fakten verneinen und sich auf den steril abgeschlossenen Bereich von Ideen beschränken. Dann ist sie aber keine Presse mehr. Oder aber sie geht auf die Straße, beschreibt, wertet mit der ihr gewohnten Unabhängigkeit, um die Stellung der Katholiken darzulegen, ohne dabei die Meinung der Hierarchie auch nur offiziös in diese Beurteilung einzubeziehen. Der freie Journalist entlastet die Kirche von Verantwortungen, die ausschließlich Bürgerpflichten sind. So verstandene journalistische Unabhängigkeit sollte eine allen bekannte ständige Voraussetzung sein. Trifft dies nicht $\mathrm{zu}$, hat es angesichts eines wirklich ernsten Falles keinen Sinn zu beteuern, die Diözesanzeitung vertrete nicht den offiziellen Standpunkt der Kirche.

Diese Forderung nach redaktionelelr Unabhängigkeit stellt das Schicksal und die Existenz der katholischen Presse für die Zukunft weit mehr in Frage, als viele anzunehmen scheinen. Kompetente Kreise fragen sich, ob heute eine offizielle katholische Presse überhaupt noch nützlich sei und ob es nicht vernünftiger wäre, im Interesse der Kirche auf diese konfessionelle Presse zu verzichten und „Zeitungen mit christlicher Grundhaltung " zu gründen. Persönlich bin ich der Auffassung, daß die konfessionelle Informationspresse auch heute noch ihre Existenzberechtigung hat, vor allem - neben anderen Gründen - weil die soziologische Entwicklung in den verschiedenen Ländern der Welt sich in einem sehr unterschiedlichen Rhy thmus vollzieht. Der kirchlichen Informationspresse im weiteren Sinne dürfte eine noch längere, gesicherte Zukunft vorausgesagt werden können, weil es sich um eine innerkirchliche Presse handelt. Diese Art von Periodika (z. B. die Bistums-Wochenzeitungen) können jedoch nur überleben, wenn sie nicht mehr "offizielle Organe der Hierarchie“ bleiben, sondern Spiegel der öffentlichen Meinung des ganzen Volkes Gottes werden. Eine solche Entwicklung setzt allerdings voraus, daß der Pluralität und dem Dialog ein sehr breiter Spielraum zugestanden wird. ${ }^{7}$

Die Kirche selbst weiß manchmal nicht, was sie mit ihrer Presse machen soll, weil sie gerade in dieser Frage nicht mit der erforderlichen Entschlossenheit handelt. So in einem südamerikanischen Staat, den ich gerade besucht habe, wo acht Diözesanblätter an politische Gruppen verpachtet wurden, weil sie in der Hand der Bischöfe zum Element der Zwietracht wurden. Zwei Verlagshäuser scheiterten an ihrer Aufgabe. Eine Anzahl Bischöfe drängte den Kardinal, den kirchlichen Hörfunksender zu verkaufen, weil dieser nicht im Stande war, weltliche Fragen zu behandeln, ohne gleichzeitig die Kirche zu kompromittieren.

Sekundärer Natur, doch sehr wichtig, ist ein weiterer Aspekt: Mit Halsstarrigkeit eine offizielle Kontrolle über das tägliche Informationsangebot ausüben, über jedes Urteil und jede Initiative entscheiden zu wollen, muß als ein Mißtrauensvotum gegenüber den kompetenten und loyalen Journalisten interpretiert werden, als wolle man ihnen nicht zugestehen, selber zu denken. Viele der fähigsten Fachkräfte, die dank ihres Kontaktes zum Volk, ihrer Ausbildung und ihres Ansehens, das sie der Kirche zur Verfügung stellen - großen Einfluß in der Offentlichkeit gewonnen haben, wechseln aus diesem Grunde zu andern Zeitungen, wo sie nicht nur besser dotiert, sondern auch angemessener respektiert werden. 
bb) Damit der Dialog mit den Bischöfen offen bleibe

Gehen wir noch etwas weiter hinsichtlich der Autonomie im Urteil. Kann der Fall eintreten, daß eine katholische Zeitung zu Recht der offiziellen Stellungnahme widerspricht? Sehr oft berechtigen durchaus annehmbare Umstände, daß eine Zeitung - die der Hierarchie gehört oder Diözesanblatt ist - eine unterschiedliche oder auch gegensätzliche Haltung zwischen Redaktion und bischöflicher Autorität unverkennbar zutage treten läßt. Es ist nicht ein Mangel an Respekt, eine unterschiedliche Meinung zur kirchlichen Autorität zu haben. Darin kann sich im Gegenteil Zusammenarbeit erweisen. Die Wörter "Dialog“ und "Dialektik“ gehören zur gleiden Familie: Die Dialektik führt zur Wahrheit nicht bloß durch die Anhäufung von übereinstimmenden Argumenten, sondern auch durch die Evidenz der Widerlegungen.

Dem mehr dogmatischen Standpunkt eines Bischofs, z. B. in Schul- und Gewerkschaftsfragen, kann der Chefredakteur eines Bistumsblattes nicht nur Argumente beifügen, sondern auch Einwände entgegenhalten: Einwände von Familienvätern, Arbeitnehmern und Arbeitgebern, welche die soziologischen und lebensnahen Aspekte der Probleme aus nächster Nähe kennen. Es kann durchaus die Folge solcher Diskussionen sein, daß der Leser bei den nächsten Wahlen nicht unbedingt so stimmt, wie dies die theologisch-moralische Argumentation des Bischofs erwarten läßt. Eine Zeirung als Vertreterin der öffentlichen Meinung und Wegbereiterin des Dialogs muß in gewissen Fällen die Rolle der "Opposition Ihrer Majestät" übernehmen. Wenn diese Opposition im Bistumsblatt nicht zum Worte kommen kann, wird sie sich anderswo Gehör verschaffen, sei es nun in einer Zeitung, die dem Einflußbereich des Bischofs entzogen ist, oder im öffentlichen Gerücht oder gar in einer Unzufriedenheit, die sehr schwer zu beseitigen ist, gerade weil sie sich nicht recht artikulieren kann. ${ }^{8}$

\section{Grenzen der Information über das innerkircbliche Leben}

\section{a) Der normale Entwicklungsprozeß}

Die Bewegung im sozialen Leben ist - wie beim Meer - die Summe zweier Prozesse: der ständigen Erneuerung (Zufluß und Verdunstung des Wassers) und des Wellengangs. In der Kirche erkennen wir die ständige Erneuerung in den sich ablösenden christlichen Generationen sowie in dem ständigen von außen kommenden Einfluß der säkularen Gesellschaft, deren Schicksal parallel mit demjenigen der Kirche läuft. In dieser dynamischen Erneuerungsbewegung gibt es Generationskonflikte, Trägheits-Momente angesichts der notwendigen Ainderungen, Rückfälle, offenen Zwist. Wir stehen vor einem Erneuerungsprozeß und - manchmal - vor einer "Selbstimpfung", deren Regelmäßigkeit von der Geschichte bestätigt wird, zyklisch früher langsamer und heute schneller. In diesem nützlichen und notwendigen Prozeß findet die Presse immer wieder Stoff für Information und kluge Kritik; denn es gehört ja zu ihrer Aufgabe, das Leben widerzuspiegeln. 
Die See kann auch hohe Wellen bringen. In jeder sozialen Gruppe gibt es eine Minderheit unruhiger Menschen, unruhig wegen der Vergangenheit, der Gegenwart oder der Zukunft: Sie sind der kleine Stein in den Schuhen der Etablierten und der Mächtigen. Sie sind die kritischen Temperamente, die Pioniere, die Propheten. Immer gab es unter ihnen, im Verlaufe der Geschichte, Schriftsteller mit beißender Feder. Heute - im Zeitalter der Massenmedien - ist es bedeutungslos, ob sie Schriftsteller sind oder nicht; denn diese Unruhe findet stets ein Echo beim Publizisten.

Der im übrigen sehr ernstzunehmenden Anfechtung fehlt auch die komische Seite nicht. Es ist erwiesen, daß bei jeder Revolution ein Teil der Revolutionäre zu Konservativen werden, sobald sie sich etwas erkämpft haben. Und das gilt auch für die Intellektuellen: Viele Forscher des Zukünftigen denken mit Wehmut an die Vergangenheit, wenn sie alte und geehrte Männer sind. Der „Berufung zum Reformator“ geht dadurch eine gewisse Glaubwürdigkeit verloren, genauso wie gewisse lärmende, leidenschaftliche, extravagante oder übertrieben jugendlich-sein-wollende Handlungen nicht zu ihrem Prestige beitragen.

Die Politik verbiegt auch das theologische Urteil. Alle Zeiten haben Theologen gekannt, die sich den Herrschenden und Etablierten als Diener zur Verfügung stellten. Theologen, die sich nicht damit begnügten, das "Maximum an Christlichem" aus einem Regime oder Regierungsprogramm herauszuholen, sondern versuchten, dieses selbst als "christliches Ideal“ darzustellen, als die glückliche Verwirklichung der katholischen Doktrin im weltlichen Bereich. Man wird immer Hoftheologen, Armeestabtheologen und Guerilla-Theologen finden; Theologen, die den Rassismus verteidigen, wie sie zur Zeit der großen Welteroberungen die Sklaverei verteidigten; Theologen, welche die Diktatur oder die gewalttätige Revolution mit bemerkenswerter Gelehrsamkeit und scharfsinnigem Geist verteidigen.

Aber sind diese meist Stoff zu Satiren bietenden menschlichen Schwächen und Verirrungen ein vollständiges Bild der heute als "Kontestation“ bezeichneten Erscheinung? Jedermann weiß, daß dies nicht der Fall ist. Es gibt berechtigte Ungeduld, legitime Proteste, Unterdrückungen, welche eine zornige Reaktion begreiflich machen. Es gibt aber auch die Sicherheit, die Wahrheit zu kennen, auch wenn man nicht im Stande ist, sie genau zu formulieren. Routine und Verstand gehen nicht immer den gleichen Weg. Die Presse soll die Freiheit genießen, die positiven wie die negativen Seiten dieser "Kontestation" widerzuspiegeln, um so der Wahrheit einen Weg zu bahnen.

c) Die Reformatoren und die Propheten

Die "Medaille“ hat eine übernatürliche Seite: nämlich die Kraft des Heiligen Geistes, der in seiner Kirche die Doktoren (die Intellektuellen des Göttlichen), aber auch die Reformatoren und die Propheten (die Anfechter des Göttlichen) erweckt. Die Reformatoren und Propheten werden manchmal als lästig empfunden. Die Geschichte lehrt uns, in welchem Ausmaß viele von ihnen unter Unverständnis und Verfolgung leiden mußten. Sie zeugt auch dafür, wie viele Jahre oder gar Generationen vorübergehen müssen, bis sich ihre Ideen schließlich doch durchsetzen. Die Kirche ist göttlichen Ursprungs und göttlichen Schicksals, aber ihre Glieder sind Menschen. Erwarten sie jetzt keine "Hintertreppengeschichten" über die Zuträgerei, die geheimen Prozesse, den mangelnden rechtlichen Schutz und die Erniedrigung der Angeklagten, 
welche schlußendlich doch wieder zu ihrem Rechte kamen. Glücklicherweise hat das offentliche Gewissen auf diesem Gebiet große Fortschritte gemacht. Gewisse Verfahren wirken abstoßend auf den Menschen von heute. Dieser Wechsel ist zum großen Teil dem öffentlichen Charakter der Massenmedien zuzuschreiben. Ich glaube fest, daß die Presse auch in der Zukunft eine wichtige Rolle in der Denunziation jeder Ungerechtigkeit und in der Forderung auf Einhaltung einer ethischen Ehrlichkeit (ohne von Nächstenliebe zu sprechen) spielen wird in allem, was die Behandlung der Menschen und Christen anbelangt. Es ist offensichtlich, daß die Denunziation der Ungerechtigkeit die Ungerechten ärgert.

\section{d) Vorsichtsmaßnahmen}

Soweit zur Pressefreiheit. Aber sie hat auch ihre Grenzen. Nicht alle, die sich als Propheten ausgeben, sind Propheten. Man kann nicht Prophetismus in Anspruch nehmen, um dem Gehorsam zu entgehen. Es ist auch kaum der Heilige Geist, der jene inspiriert, die geistigen Zündstoff an das Fundament der Kirche legen. Die zur Kritik befugten Leute - auch die Journalisten - genießen diese Freiheit, um der Wahrheit und der Gerechtigkeit zu dienen und nicht um ihre Unzufriedenheit und ihre schlechte Laune in die Offentlichkeit zu tragen.

Man kann die Frage stellen, ob die katholische Presse und die katholischen Journalisten immer eine den Umständen entsprechende, ausgewogene Haltung einnahmen, um die "Kontestations"-Erscheinungen in ihrer wirklichen Bedeutung wiederzugeben. $\mathrm{Ob}$ sie in einigen dieser Erscheinungen jenen vorhandenen, jedoch in dem turbulenten Vorgehen kaum durchschimmernden Fundus an gerechter Sache erkannten; oder ob sie eine Sache nur deshalb ins gute Licht stellten, weil die gute Absicht der Protagonisten ihnen sympathisch war. Die Massenmedien brauchen eine gerechte Forderung nur totzuschweigen, wenn sie deren Scheitern erreichen wollen. Umgekehrt genügt es, wenn ein Mann ohne den geringsten gesunden Menschenverstand zur Verbreitung seiner Meinung über den für Millionen hörbaren Lautsprecher verfügt, damit er künstlich den "Status" eines Leaders erhält, auch wenn die gleiche Presse ihn eine Woche später schon wieder ignoriert und von der Tribüne stürzt. - Der Journalist darf nicht verschweigen, was die schweigende Mehrheit denkt, nur weil eine Minderheit sich mit größter Lautstärke vernehmen läßt. Leider wird er den Propheten - und zu dessen Nachteil - nicht immer vom Exhibitionisten unterscheiden können.

Reformen bedürfen zudem, wie jeder gute Wein, eines Reifungsprozesses. Die Pioniere sind zu bewundern. Angesichts des Gewinnes, den sie der Kirche durch ihren Einsatz für die Wahrheit und das Gute verschaffen, indem sie Doktrinen korrigieren, welche ehrwürdig scheinen, während sie nur alt sind, verdienen sie Nachsicht gegenüber ihren eventuell möglichen Irrtümern. Jeder, der in der Dunkelheit einen Weg sucht, läuft Gefahr, in den Abgrund zu fallen. Früher gab es noch eine natürliche Gärungsund Ruheperiode vor der Reife. Diese natürliche Reifung wird durch die Massenmedien verkürzt. Den Kommunikationsmitteln von heute kommt das Verdienst zu, in wenigen Jahren eine Entwicklung beschleunigt zu haben, die vorher Jahrhunderte dauerte. Aber gleichzeitig sind sie zum guten Teil verantwortlich für die derzeitige äußere Krise der Kirche.

\section{e) Das Verhältnis von wahr und gut}

Die Begriffe "wahr" und "gut" sind - logischerweise - verschieden. Vom Wesen her müssen die Begriffe des "Wahren" und "Guten" übereinstimmen. Moralisch ist 
die Wahrheit ohne das Gute verachtenswert. Die Verleumdung ist die Wahrheit ohne das Gute; der Skandal desgleichen; viele Rebellionen und Schismen waren das Ergebnis des Wahren ohne das Gute.

Der Journalist bewegt sich nicht nur unter reifen Menschen, sondern auch unter Kindern, Jugendlichen, Einfältigen und Schwachen. Mancher wird verwirrt durch die äußerlichen Gegensätze unter Bischöfen, das äußerliche In-Frage-Stellen der päpstlichen Autorität oder durch die äußerliche Berufs-Aufgabe von Priestern. Vorsicht und Nächstenliebe sollten den Journalisten leiten. In den Zeitungen mit breiter Streuung muß das Wohl der Kirche immer den ersten Rang einnehmen - auch in Informationsfragen - über dem Informationsanspruch einer gebildeten Minderheit, der man in einer speziell für sie bestimmten Publikation ein spezialisierteres Informationsangebot machen kann.

Verschiedene Einwände sind möglich: Unter dem Vorwand, den Schwachen Ärgernis zu geben, kann ein Journalist mundtot gemacht werden, obwohl er den Skandal der Pharisäer aufdecken wollte.

Der zweite: Es gibt Mächtige, die nicht „das Wohl“, sondern nur „ihr Wohl“ verteidigen und es als unklug ansehen, andere als ihre eigenen Ideen zu vertreten.

Der dritte: Es gibt Gruppen, die zur journalistischen Vorsicht mahnen, weil das „einfache Volk“ zu wenig gebildet sei. Haben sie je etwas unternommen, um ihm $\mathrm{zu}$ dieser Bildung $\mathrm{zu}$ verhelfen, wo doch gerade die Information ein rasch wirkendes Mittel wäre, um der kollektiven Ignoranz beizukommen?

Die vierte: Wie ich schon sagte, ist das Schweigen der katholischen Journalisten nutzlos, wenn die anderen sprechen.

Die gesamten Ausführungen könnte man zusammenfassen im Kriterium: Informiert mit Liebe.

\section{Anmerkungen:}

1. Laut "Correspondance de la Presse ${ }^{\alpha}$ (Paris, Nr. 5103 vom 24. November 1969, S. 17) sind 60 bis 91 v. H. der Totaleinnahmen der britischen Presse Einnahmen aus Anzeigen. In andern Ländern ist der Anteil der Inserateneinnahmen geringer. In jedem Fall liegt er jedoch weit über dem Verkaufserlös für die einzelne Ausgabe.

2. Wir möchten hervorheben, daß in der Weltpresse, von "Life ${ }^{\alpha}$ und "Paris Match" bis zu den großen Tageszeitungen "The New York Times" und "Neue Zürcher Zeitung" wie auch in den Hörfunk- und TV-Sendungen - mit ihren Hunderten von Millionen Lesern, Zuhörern und Zuschauern - der katholische Leser vom nichtkathoilschen nicht durch eine willkürliche Linie getrennt werden darf. Namen wie Defregger, Illich, Schillebeeckx, Isolotto, Boquen, Cuernavaca sind durch die Setzmaschinen der fünf Kontinente gegangen. Hat es dann noch einen Sinn, daß Diözesanblätter diese Namen verschweigen?

3. Schreiben des Päpstlichen Staatssekretariates an die 53. „Semaine Sociale de France, Juli 1966.

4. "Pacem in terris", Nr. 12. - Wir beschränken uns hier auf einige der wichtigsten Ideen. Zeugnisse des päpstlichen Lehramtes sind zur Genüge, sowohl für die Zeit vor wie nach dem Vatikanum II, vorhanden. Eines der bedeutendsten, das man als klassisch bezeichnen kann, betrifft die öffentliche Meinung in der Kirche, nämlich die Botschaft Pius' XII. vom 18. 2. 1950 an die Katholische Weltunion der Presse. Vgl. auch Jesús Iribarren u. a.: "Cinco grandes Mensajes" ("Biblioteca de Autores Cristianos"), Madrid 1968, 382 S. Ders.: „El derecho a la verdad: doctrina de la Iglesia sobre prensa, radio y televisión“, Madrid 1968, 86 u. 504 S. 
5. Diese Frage habe ich in dem „Rundschreiben aus dem Sekretariat der Union Catholique Internationale de la Presse“, Paris, Nr. 4 vom 27. Januar 1969, aufgeworfen: "Hat die Regierung der Kirche nicht das Recht, Aussprachen im geschlossenen Kreis zu veranstalten wie irgendeine staatliche Regierung, eine Stabsgruppe, ein Verwaltungsrat?" Ein Ja auf diese Frage scheint mir klar zu sein, und es gilt auch für die untergeordneten Beratungsund Beschlußinstanzen. Für Leute mit gesundem Menschenverstand braucht es hier keine langen Überlegungen.

6. Vgl. José Luiz Martin Descalzo: La communication du Sacré et l'Information sur l'Eglise, in: "Journalistes Catholiques", Paris, Nr. $46 / 47$ von Juli/Oktober 1969. - Emile Poulat: „Intégrisme et Catholicisme intégral“ (Casterman), Paris 1969.

7. Vgl. Federazione Italiana Settimanali Cattolici (Hrsg.): Documento programmatico per i settimanali Cattolici, in: ${ }_{n}$ Informations de la F.I.S.C.“, Rom, Nr. 4 vom 26. Oktober 1969 und in: "Journalistes Catholiques", Paris, Nr. 48 von Oktober/Dezember 1969.

8. In „Journalistes Catholiques“, Paris, Nr. 35 von September/Oktober 1967, ist dieses Problem ausführlich behandelt worden am Beispiel einer Polemik in "Münchener Katholische Kirchenzeitung ": „Die Bistumszeitung: Eine ,Einbahnstraße?"

\section{S U M M A R Y}

The World Congress of the International Catholic Press Union (UCIP) to be held in Luxembourg 1971 will handle as principal theme the problem of the public opinion within the Church. This article of the UCIP General Secretary Jesús Iribarren deals with the question of the liberty of the press in the Catholic sphere and shall serve for the preparation of this meeting. The author presupposes that the obligation of information is an essential component of the press and that free flow of information answers to the need of the society. Also the Church has taken into consideration these facts in the communication work. However, limits of the free expression of opinion are given by dogma and the leading authorities. On the other hand the clerical press would make itself dubious if it would depend on the directives of the hierarchy also in the treatment of general questions of life and society. The press even has the duty to exonorate the official Church from responsibility in such questions.

\section{R E S U M E N}

El Congreso Mundial de 1971 de la „Union Catholique Internationale de la Presse (UCIP) en Luxemburgo tratará como tema principal el problema de la opinión pública en la Iglesia. La contribución presente del Secretario General de la UCIP, Jesús Iribarren, a la cuestión de la libertad de la Prensa en el campo católico debe servir como preparación para esta reunión. El autor parte de que el deber de informar es un criterio de la prensa y la libertad de la información corresponde a una necesidad de la sociedad. La Iglesia también ha de tener en cuenta estos hechos en su trabajo de comunicación. Sin duda por dentro la Iglesia hay límites de la manifestación libre de opiniones, fijados por dogma y autoridad de enseñanza. Por otra parte la prensa eclesiástica misma se pondría en duda, si quedara dependiente de los órdenes de la jerarquía también referente al tratamiento de cuestiones generales de vida y sociedad. Es obligada aún a eximir a la Iglesia oficial de la responsabilidad en estas cuestiones. 